\title{
Water as catalyst and solvent: tetrahydropyranylation of alcohols in an aqueous medium
}

\author{
Veerababurao Kavala, Akshaya Kumar Samal, and Bhisma K. Patel* \\ Department of Chemistry, Indian Institute of Technology Guwahati, Guwahati-781 039, India \\ E-mail: patel@iitg.ernet.in
}

(received 20 Aug 04; accepted 13 Oct 04; published on the web 26 Oct 04)

\begin{abstract}
Water is found to catalyze the tetrahydropyranylation of alcohols at elevated temperature. Interestingly, tetrahydropyranylation of alcohols works under a wide range of $\mathrm{pH}, 6.5$ to 2, and does not work beyond $\mathrm{pH} 7.5$ in an aqueous medium. Hydrophobic interactions between the substrate alcohol and dihydropyran ether (DHP) and favorable pKa's of water, alcohols, and protonated hydroxyl and protonated ethereal functions are the driving forces for the reaction. Thus the reaction, which is carried out in anhydrous aprotic solvents, can be carried out in aqueous medium in an environmentally benign way.
\end{abstract}

Keywords: Protection, aqueous medium, tetrahydropyranylation, chemoselectivity

\section{Introduction}

Aqueous mediated reactions have received considerable attention in organic synthesis due to environmental safety reasons. Water is the cheapest, most abundant and non-toxic chemical in Nature. It has unique physical and chemical properties such as a high dielectric constant and cohesive energy density compared to organic solvents. It has also special effects on reactions arising from inter and intramolecular non-covalent interactions leading to novel solvation and assembly processes. Large numbers of organic reactions have been performed successfully utilizing water as the reaction medium, and several books and reviews have been devoted to such a use. ${ }^{1}$ Thus, development of an efficient and convenient synthetic methodology in water is an important subject in these environmentally conscious days. Recently, we have reported acylation of amines in an aqueous medium. ${ }^{2}$

\section{Results and Discussion}

The tetrahydropyranyl ether is one of the most frequently used protecting groups for alcohols employed during multi-step organic synthesis. ${ }^{3}$ Most of the reported methods use acidic reagents in an aprotic solvent such as $\mathrm{CH}_{2} \mathrm{Cl}_{2}$, THF, acetone and toluene. ${ }^{4}$ At times their formation has 
been carried out in ionic liquids. ${ }^{5}$ Although these methods are suitable for many synthetic conditions, some of these methods suffer from use of excess amounts and/or toxic catalysts, volatile organic solvents and large amounts of solid supports which ultimately result in the generation of considerable amounts of toxic wastes. Tetrahydropyranylation is usually performed in anhydrous aprotic organic solvents because of the longer reaction time and poor yields of products obtained in the presence of water. Based on the pKa's of water (15.74), alcohols $(\sim 16.5)$, protonated hydroxyl of 2-hydroxytetrahydropyran ( -2) and protonated ethereal functions $(\sim-6)$, we speculate that pyranylation of alcohols should occur in an aqueous medium and, due to an unfavorable pKa, phenol should not undergo pyranylation in water. 2Hydroxytetrahydropyran is formed in all reactions involving dihydropyran (DHP) and water. The hydroxyl group of 2-hydroxytetrahydropyran is more likely to be protonated compared to the ethereal oxygen. An oxycarbenium species could be generated due to protonation of the hydroxyl group followed by water elimination, which should react with alcohol giving rise to the corresponding THP ether. The attack of water occurs but it is unproductive and regenerates the oxycarbenium species via a hemiacetal while the attack of the alcohol produces the acetal. The latter should be favored because the hydroxyl group having a lower pKa will leave preferentially over the alkoxide, driving the reaction in favor of product formation as shown in Scheme 1.

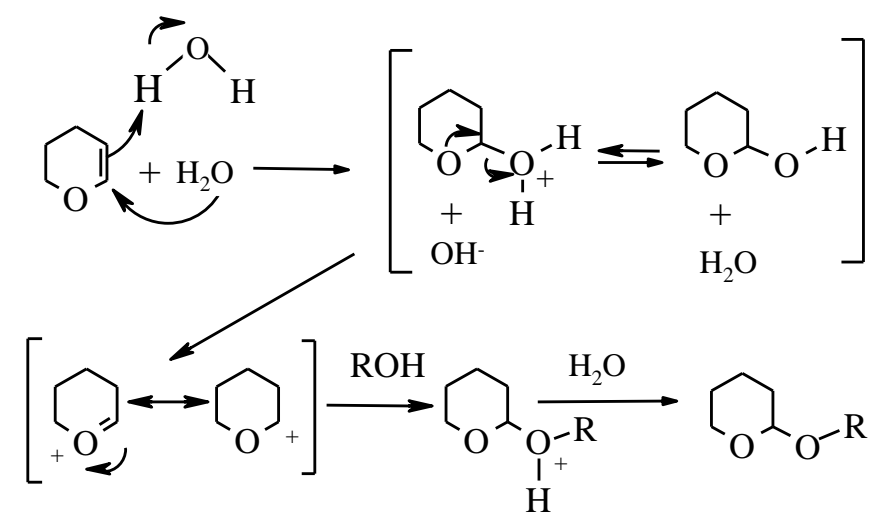

Scheme 1. Mechanism of formation of THP ether in water.

With this assumption, when 3-phenyl-1-propanol 2 (5 mmol) was treated with DHP $(7.5 \mathrm{mmol})$ in water $(5 \mathrm{ml}), 60 \%$ of the product was formed after $24 \mathrm{~h}$ at room temperature. However, ca. $60 \%$ conversion was achieved in $7 \mathrm{~h}$ by performing the reaction at reflux temperature. Alcohol and DHP formed a heterogeneous reaction mixture in water. In order to make the reaction homogeneous with the hope of accelerating the reaction, phase transfer reagents such as tetrabutylammonium bromide ( $c a .50 \mathrm{mg}$ ) were added to the medium. Unfortunately, the percentage conversion was a mere $50 \%$ after $10 \mathrm{~h}$, even at reflux temperature. When the reaction was repeated in a saturated solution of sodium chloride under reflux conditions, the yield improved to $c a$. $80 \%$ within $7 \mathrm{~h}$. Thus, hydrophobic interactions between the alcohol and the DHP unit are essential for the reaction leading to product formation. The $\mathrm{pH}$ of the medium progressively decreased from ca. 6.8 at the beginning of the reaction to $c a .4 .3$ after the completion of the reaction when 3-phenyl-1-propanol 2 was used as the substrate. This prompted 
us to investigate the optimum $\mathrm{pH}$ conditions of the reaction medium required for the pyranylation of alcohol. Pyranylation was performed under reflux at different $\mathrm{pH}$ (1 M buffer), saturated with $\mathrm{NaCl}$. The results are summarized in Table 1.

Table 1. Tetrahydropyranylation of 3-phenyl-1-propanol 2 in different buffers

\begin{tabular}{|c|c|c|c|}
\hline $\mathrm{pH}$ of the Solution & Time $(\mathrm{h})$ & Conversion $(\%)^{\mathrm{a}}$ & Types of Buffer Used \\
\hline 8 & 15 & Nil & $\mathrm{Na}_{2} \mathrm{HPO}_{4}+\mathrm{NaH}_{2} \mathrm{PO}_{4}$ \\
\hline 7.5 & 15 & $10 \%$ & $\mathrm{Na}_{2} \mathrm{HPO}_{4}+\mathrm{NaH}_{2} \mathrm{PO}_{4}$ \\
\hline 7 & 15 & $20 \%$ & $\mathrm{Na}_{2} \mathrm{HPO}_{4}+\mathrm{NaH}_{2} \mathrm{PO}_{4}$ \\
\hline 6.5 & 15 & $72 \%$ & $\mathrm{Na}_{2} \mathrm{HPO}_{4}+\mathrm{NaH}_{2} \mathrm{PO}_{4}$ \\
\hline 6 & 15 & $80 \%$ & $\mathrm{Na}_{2} \mathrm{HPO}_{4}+\mathrm{NaH}_{2} \mathrm{PO}_{4}$ \\
\hline 5.5 & 10 & $>95 \%$ & $\mathrm{Na}_{2} \mathrm{HPO}_{4}+\mathrm{NaH}_{2} \mathrm{PO}_{4}$ \\
\hline 5 & 8 & $>95 \%$ & $\mathrm{Na}_{2} \mathrm{HPO}_{4}+\mathrm{NaH}_{2} \mathrm{PO}_{4}$ \\
\hline 4.5 & 7 & $>95 \%$ & $\mathrm{Na}_{2} \mathrm{HPO}_{4}+\mathrm{NaH}_{2} \mathrm{PO}_{4}$ \\
\hline 4 & 5 & $>95 \%$ & Sodium citrate + Citric acid \\
\hline 3.5 & 4.5 & $>95 \%$ & Sodium citrate + Citric acid \\
\hline 3 & 3 & $>95 \%$ & Sodium citrate + Citric acid \\
\hline 2 & 1 & $>95 \%$ & Sodium citrate + Citric acid \\
\hline
\end{tabular}

${ }^{\mathrm{a}} \mathrm{GC}$ yields.

It is interesting to note that the reaction worked from $\mathrm{pH} 7.5$ down to $\mathrm{pH} 2$. The process is more facile at lower $\mathrm{pH}$ and worked even in $10 \%$ aqueous $\mathrm{HCl}$ giving $90 \%$ yield of the product after $2.5 \mathrm{~h}$. It seems that under acidic conditions the oxycarbenium species is more susceptible to attack by alcohol than water (Scheme 1). There are several reports of deprotection of THP ethers $^{3}$ in an acidic medium and in almost all of these cases methanol or ethanol is used as the co-solvent, possibly because a large excess of the alcohol shifts the equilibrium with concurrent formation of 2-methoxytetrahydropyran via a transacetalization process. When the THP ether of 3-phenyl-1-propanol 2a (1 mmol) was treated with $10 \%$ aq. $\mathrm{HCl}(2 \mathrm{~mL})$ and refluxed for $2 \mathrm{~h}$, less than $5 \%$ of the product was deprotected. However, more than $90 \%$ deprotection could be achieved after $2 \mathrm{~h}$ under identical conditions when methanol $(2 \mathrm{~mL})$ was added to the medium. When pyranylation of 3-phenyl-1-propanol $2(1 \mathrm{mmol})$ was performed under identical conditions at $\mathrm{pH} 2(1 \mathrm{M}$ buffer $)$ in the presence of methanol $(2 \mathrm{~mL})$, only a trace of pyranylated product $(<$ $5 \%$ ) could be seen, further supporting the facile formation of 2-methoxytetrahydropyran and the role of methanol during the depyranylation process (Scheme 2). 


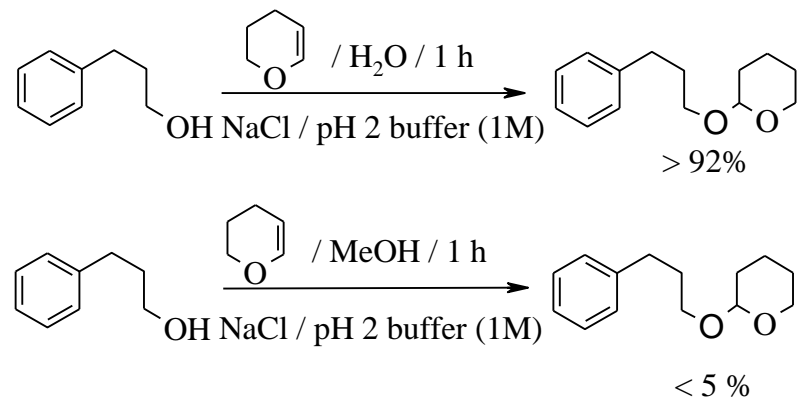

Scheme 2. Pyranylation in different solvents.

Mechanistically, it is possible to suggest other reasonable pathways, as for instance the addition of the alcohol to the aldehyde group of the open-chain tautomer of 2hydroxytetrahydropyran followed by protonation of the hemiacetal $\mathrm{OH}$, loss of water, intramolecular acetalisation followed by deprotonation. This possibility can be ruled out because IR and ${ }^{1} \mathrm{H}$ NMR analysis of 2-hydroxytetrahydropyran showed less than $1 \%$ of the open chain tautomer. Similar to reducing sugars it may contain $<0.01 \%$ of the aldehydic form, however unlike glucose, 2-hydroxytetrahydropyran failed to respond to both Tollen's and Fehling's tests. In another experiment, when 2-hydroxytetrahydropyran $(5 \mathrm{mmol})$ and 3-phenyl-1-propanol 2a (2 mmol) were reacted in a pH 2 buffer $(1 \mathrm{M}, 2 \mathrm{~mL})$ saturated with $\mathrm{NaCl}$ and refluxed for $2 \mathrm{~h}$, more than $95 \%$ of the pyranylated product could be obtained, further supporting the intermediacy of 2hydroxytetrahydropyran (Scheme 3).

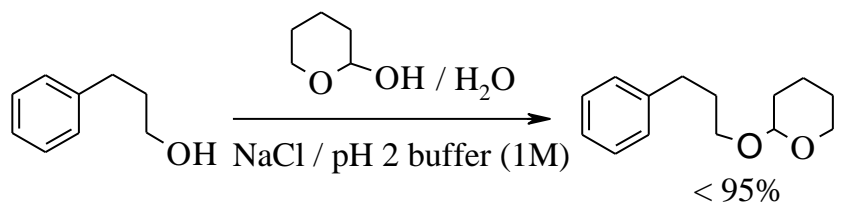

Scheme 3. Pyranylation of 3-phenyl-1-propanol 2 with 2-hydroxytetrahydropyran.

The reaction works even in pure water under non buffered conditions, giving a moderate yield of the product, but the $\mathrm{pH}$ of the medium changes depending on the nature of the substrate. Therefore, we decided to perform the reaction under buffered conditions. In spite of the shorter reaction time at lower $\mathrm{pH}$ we decided to perform the reaction at $\mathrm{pH} 5$ since many acid sensitive groups are expected to survive at this $\mathrm{pH}$. Thus, in a typical reaction, alcohol $(5 \mathrm{mmol})$, DHP (7.5 mmol), in $1 \mathrm{M}$ phosphate buffer $(5 \mathrm{~mL})$ saturated with $\mathrm{NaCl}$ was refluxed for a specific period of time as shown in Table 2. 
Table 2. Tetrahydropyranylation ${ }^{\mathrm{a}}$ of alcohol in phosphate buffer $\mathrm{pH} 5$

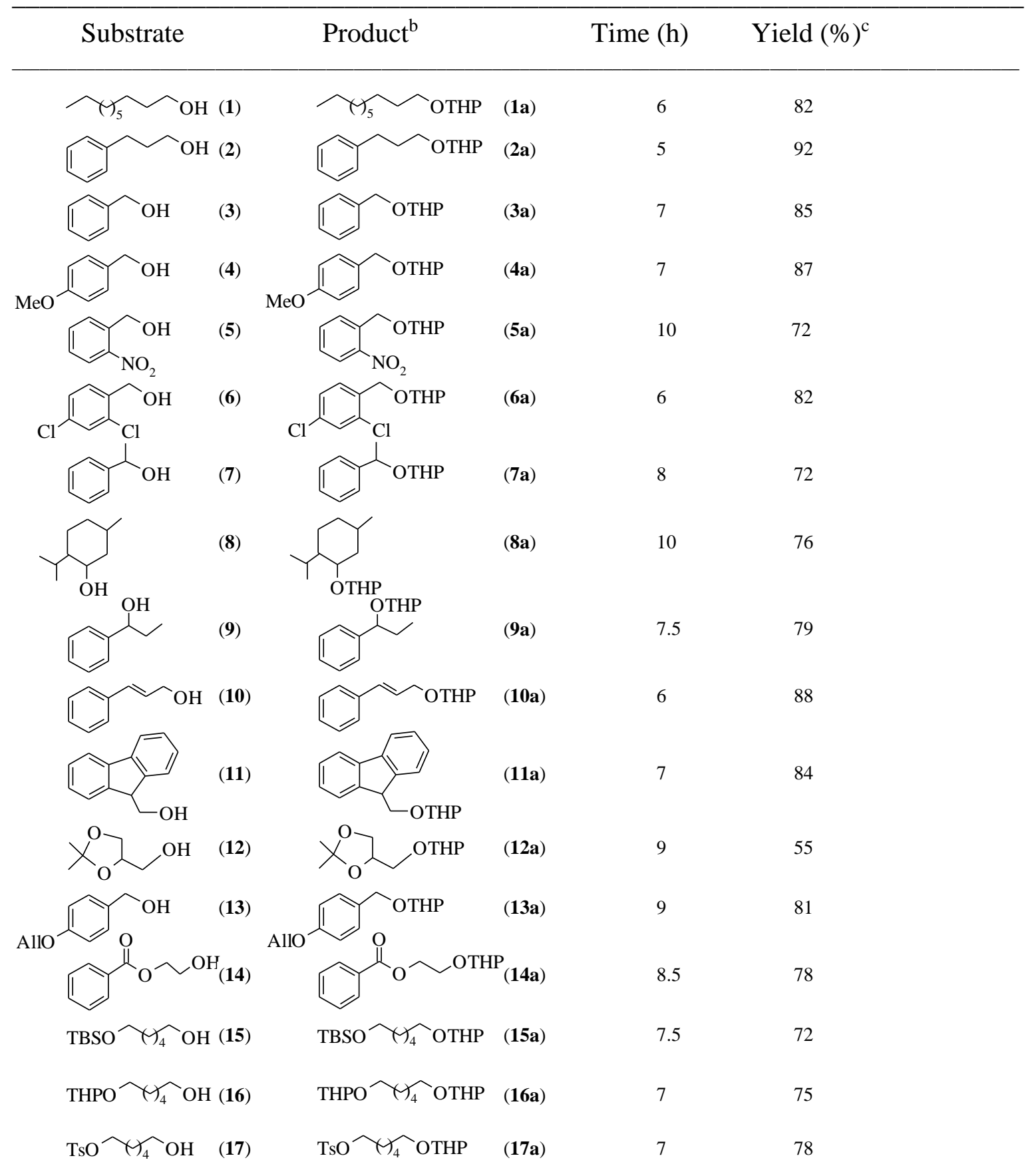

${ }^{a}$ Reactions were monitored by TLC. ${ }^{b}$ Confirmed by comparison with IR, ${ }^{1} \mathrm{H}$ NMR of the authentic sample. ${ }^{\mathrm{C}}$ Isolated yields.

Pyranylation of several alcohols such as primary, benzylic and secondary was achieved in good to excellent yields via this procedure as shown in Table 2. In all the cases examined the reactions were nearly complete, however isolated yields were 10-15\% less. As evident from Table 
2, acid sensitive protecting groups such as THP, TBS, TBDPS and isopropylidine survived these conditions. Thus, the present method is mild enough to tolerate a wide range of functional groups. As shown in Table 1, depending on the sensitivity of the other protecting group present in the substrate, this reaction can be performed from $\mathrm{pH}$ 6.5-2. Phenol, which has a lower $\mathrm{pKa}$ than water, is a better leaving group; hence the method was not successful for pyranylation of phenols in the entire range of $\mathrm{pH}$ tested. One important feature of this method is the chemoselective mono tetrahydropyranylation of symmetrical 1,n-diols 18 and 19 and unsymmetrical diol 20 (Table 3), a transformation that is difficult to accomplish via conventional methods. Only a few methods have been reported in the literature. ${ }^{4 \mathrm{~m}-\mathrm{s}, 5 \mathrm{a}-\mathrm{b}} \mathrm{We}$ assume that the selectivity of the mono tetrahydropyranylation arises because, after the monopyranylation, the other hydroxyl group points away from the hydrophobic pockets formed by the DHP and alcohol. A primary alcolol has been selectively pyranylated in the presence of a secondary alcohol $\mathbf{2 0}$ and a phenolic hydroxyl group 21.

Table 3. Tetrahydropyranylation ${ }^{\mathrm{a}}$ of alcohol in water

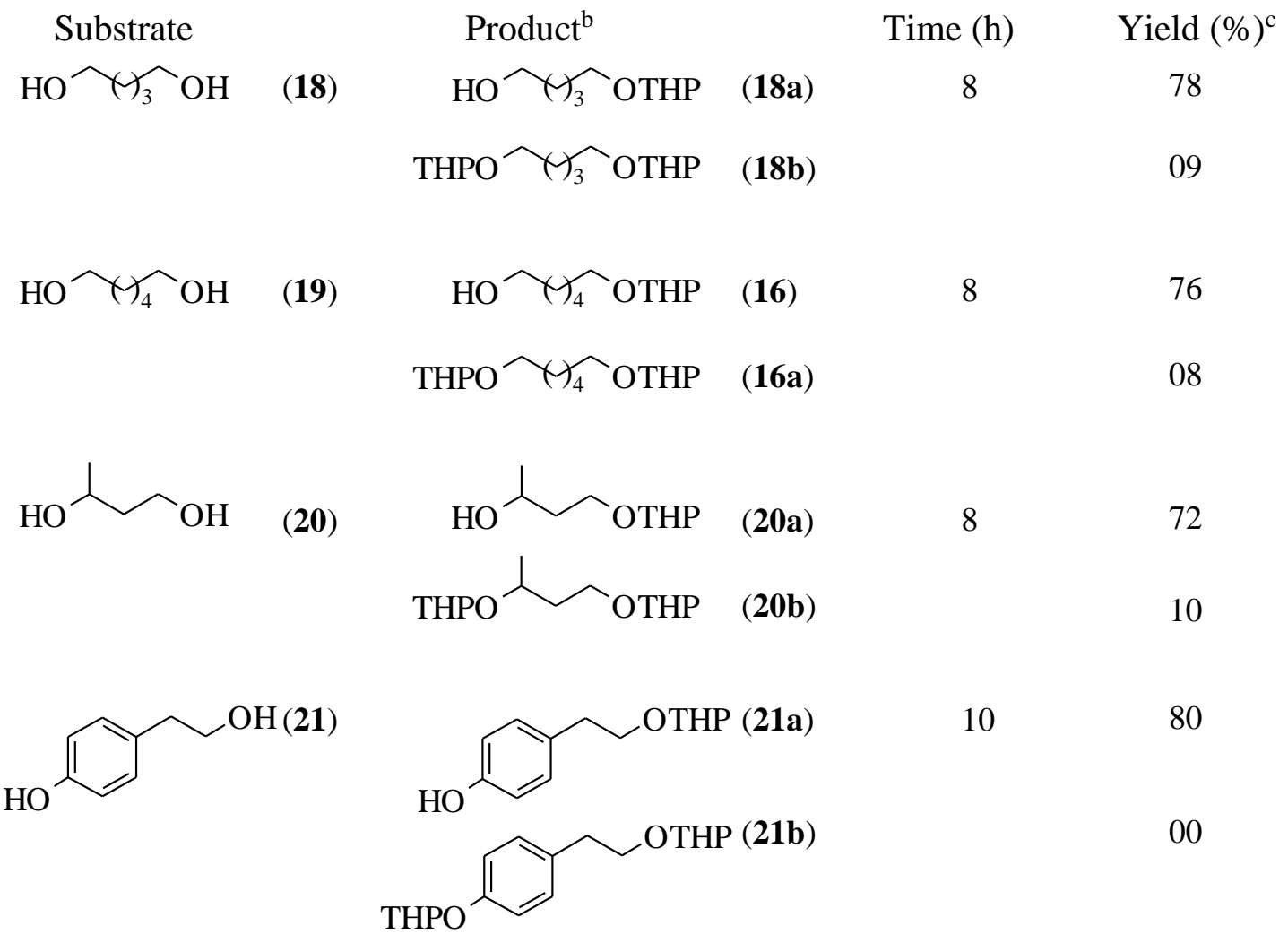

${ }^{a}$ Reactions were monitored by TLC. ${ }^{\mathrm{b}}$ Confirmed by comparison with IR, ${ }^{1} \mathrm{H}$ NMR of the authentic sample. ${ }^{\mathrm{c}}$ Isolated yields. 


\section{Conclusions}

In conclusion, this manuscript describes a method for pyranylation in water that takes advantage of the pKa's of different functional groups and the reaction medium. Thus the reaction, which is typically carried out in an anhydrous aprotic solvent, can be carried out in water in an environmentally benign way. The procedure works over a broad $\mathrm{pH}$ range and even without an acid catalyst. Mild reaction conditions, simplicity of the procedure, general applicability for a wide range of alcohols and selective mono-tetrahydropyranylation of diols are other significant advantages over many existing procedures.

\section{Experimental Section}

General experimental procedure. To a mixture of alcohol $(5 \mathrm{mmol})$ and 3,4-dihydro- $2 \mathrm{H}$-pyran ( $7.5 \mathrm{mmol}$ ) was added $1 \mathrm{M} \mathrm{Na}_{2} \mathrm{HPO}_{4}+\mathrm{NaH}_{2} \mathrm{PO}_{4}$ buffer $(5 \mathrm{~mL}$ ) saturated with $\mathrm{NaCl}$. The reaction was refluxed in a oil bath and the progress of the reaction was monitored by TLC. After the specific time, the reaction mixture was extracted with ethyl acetate $(2 \times 25 \mathrm{~mL})$. The combined organic extracts were dried with anhydrous $\mathrm{Na}_{2} \mathrm{SO}_{4}$ and the solvent was removed in a rotary evaporator under reduced pressure. The crude product was purified by passing through a short column of silica gel to yield the pure product, which was identified by its $\mathrm{NMR}^{6}$ and IR spectra and GC pattern, and GC co-injection with authentic samples prepared by known methods.

\section{Acknowledgements}

B.K.P. acknowledges the support of this research from DST New Delhi SP/S1/G-28/98 and CSIR 01(1688)/00/EMR-II and A.K.S. acknowledges the financial support to the CSIR and V.R.K to the institute. Thanks are due to CIF IIT Guwahati, RSIC, Lucknow and IIT Kanpur for providing NMR spectra.

\section{$\underline{\text { Supplementary Material Available }}$}

\section{References and Notes}

1. (a) Breslow, R. Acc. Chem. Res. 1991, 24, 159. (b) Li, C. -J. Chem. Rev. 1993, 93, 2023. (c) Herrmann, W. A.; Kohlpaintner, C. W. Angew. Chem., Int. Ed. 1993, 32, 1524. (d) Lubineau, A.; Auge, J.; Queneau, Y. Synthesis 1994, 741. (e) Li, C. -J. Tetrahedron 1996, 52, 5643. (f) Kobayashi, S. Synlett 1994, 689. (g) Lindström, U. M. Chem. Rev. 2002, 102, 2751. (h) Shu, K.; Kei, M. Acc. Chem. Res. 2002, 35, 209. (i) Okuhara, T. Chem. Rev. 2002, 102, 3641. (j) Pae, A. N. Cho, Y. S. Current Organic Chemistry 2002, 6, 715.

2. (a) Naik, S.; Bhattacharjya, G.; Talukdar, B.; Patel. B. K. Eur. J. Org. Chem. 2004, 1245. (b) Naik, S.; Bhattacharjya, G.; Kavala, V. R.; Patel B. K. ARKIVOC 2004, 55. 
3. (a) Greene, T. W.; Wuts, P. G. M. Protective Groups in Organic Synthesis; $3^{\text {rd }}$ Edn; John Wiley \& Sons: New York, 1999. (b) Kocieński, P. J. Protecting Groups; Georg Thieme Verlag: New York, 1994. (c) Jarowicki, K.; Kocieński, P. J. Chem. Soc., Perkin Trans.1 2000, 2495.

4. (a) Babu, B. S.; Balasubramanian, K. K. Synlett 1999, 1261. (b) Curini, M.; Epifano, F.; Marcotullio, M. C.; Rosati, O.; Costantino, U. Tetrahedron Lett. 1998, 38, 8159. (c) Babu, B. S.; Balasubramanian, K. K. Tetrahedron Lett. 1998, 39, 9287. (d) Mineno, T. Tetrahedron Lett. 2002, 43, 7975. (e) Romanelli, G. P.; Baronetti, G.; Thomas, H. J.; Autino, J. C. Tetrahedron Lett. 2002, 43, 7589. (f) Stephens, J. R.; Butler, P. L.; Clow, C. H.; Oswald, M. C.; Smith, R. C.; Mohan, R. S. Eur. J. Org. Chem. 2003, 3827. (g) Ravindranathan, N.; Ramesh, C.; Das, B. Synlett 2001, 1777. (h) Nishiguchi, T.; Hayakawa, S.; Hirasaka, Y.; Saitoh, M. Tetrahedron Lett. 2000, 41, 9843. (i) Pachamuthu, K.; Vankar, Y. D. J. Org. Chem. 2001, 66, 7511. (j) Ranu, B. C.; Saha, M. J. Org. Chem. 1994, 59, 8269. (k) Hon, Y.S.; Lee, C.-F. Tetrahedron Lett. 1999, 40, 2389. (1) Naik, S.; Gopinath, R.; Patel, B. K. Tetrahedron Lett. 2001, 42, 7679. (m) Nishiguchi, T.; Kawamine, K. Chem. Commun. 1990, 1766. (n) Khan, A. T.; Mondal, E.; Borah, B. M.; Ghosh, S. Eur. J. Org.Chem. 2003, 4113. (o) Namboodiri, V. V.; Varma, R. S. Tetrahedron Lett. 2002, 43, 1143. (p) Karimi, B.; Maleki, J. Tetrahedron Lett. 2002, 43, 5353. (q) Kumar, H. M. S.; Reddy, B. V. S.; Reddy, E. J.; Yadav, J. S. Chem. Lett. 1999, 857. (r) Nishiguchi, T.; Fujisaki, S.; Kuroda, M.; Kajisaki, K.; Saitoh, M. J. Org. Chem. 1998, 63, 8183. (s) Nishiguchi, T.; Kawamine, K.; Ohstuka, T. J. Chem. Soc., Perkin Trans 1 1992, 153. (t) Deka, N.; Sarma, J. C. J. Org. Chem. 2001, 66, 1947.

5. (a) Namboodiri, V. V.; Varma, R. S. Chem. Comm. 2002, 342. (b) Yadav, J. S.; Reddy, B. V. S.; Gnaneshwar, D. New. J. Chem. 2003, 27, 202. (c) Branco, L. C.; Afonso, C. A. M. Tetrahedron 2001, 57, 4405.

\section{Spectroscopic data of compounds}

1a. ${ }^{1} \mathrm{H}$ NMR (400 MHz, $\left.\mathrm{CDCl}_{3}\right) \delta 0.90$ (t, 3H, $J=7.2 \mathrm{~Hz}$ ), 1.20-1.40 (brs, $\left.14 \mathrm{H}\right), 1.50-1.69$ $(\mathrm{m}, 6 \mathrm{H}), 1.7(\mathrm{~m}, 1 \mathrm{H}), 1.80(\mathrm{~m}, 1 \mathrm{H}), 3.35(\mathrm{~m}, 1 \mathrm{H}), 3.50(\mathrm{~m}, 1 \mathrm{H}), 3.75(\mathrm{~m}, 1 \mathrm{H}), 3.85(\mathrm{~m}, 1 \mathrm{H})$, $4.60(\mathrm{~m}, 1 \mathrm{H}) .{ }^{13} \mathrm{C} \mathrm{NMR}\left(100 \mathrm{MHz}, \mathrm{CDCl}_{3}\right) \delta 14.5,20.1,23.1,25.9,26.6,29.7,29.9,29.98$, 30.0, 30.2, 31.1, 32.3, 62.6, 68.0, 99.0.

2a. ${ }^{1} \mathrm{H}$ NMR (400 MHz, $\left.\mathrm{CDCl}_{3}\right) \delta 1.48-1.96$ (m, 8H), 2.69 (m, 2H), 3.35-3.49 (m, 2H), 3.72$3.85(\mathrm{~m}, 2 \mathrm{H}), 4.56(\mathrm{~m}, 1 \mathrm{H}), 7.23(\mathrm{~m}, 5 \mathrm{H}) .{ }^{13} \mathrm{C} \mathrm{NMR}\left(100 \mathrm{MHz}, \mathrm{CDCl}_{3}\right) \delta 19.5,25.4,30.6$, 31.2, 32.4, 62.1, 66.7, 98.7, 125.6, 128.1, 128.3, 141.9.

3a. ${ }^{1} \mathrm{H}$ NMR $\left(400 \mathrm{MHz} \mathrm{CDCl}_{3}\right) \delta 1.48-1.90(\mathrm{~m}, 6 \mathrm{H}), 3.52(\mathrm{~m}, 1 \mathrm{H}), 3.89(\mathrm{~m}, 1 \mathrm{H}), 4.42(\mathrm{~d}$, $1 \mathrm{H}, J=11.6 \mathrm{~Hz}), 4.70(\mathrm{~m}, 1 \mathrm{H}), 4.80(\mathrm{~d}, 1 \mathrm{H}, J=12.0 \mathrm{~Hz}) 7.25(\mathrm{~m}, 5 \mathrm{H}) .{ }^{13} \mathrm{C} \mathrm{NMR}(100 \mathrm{M}$ $\left.\mathrm{Hz}, \mathrm{CDCl}_{3}\right) \delta 19.8,25.9,31.0,62.4,69.1,97.9,127.7,128.0,128.5,138.5$.

4a. ${ }^{1} \mathrm{H}$ NMR (400 MHz, $\left.\mathrm{CDCl}_{3}\right) \delta 1.40-1.91(\mathrm{~m}, 6 \mathrm{H}), 3.50(\mathrm{~m}, 1 \mathrm{H}), 3.76(\mathrm{~s}, 3 \mathrm{H}), 3.90(\mathrm{~m}$, $1 \mathrm{H}), 4.42(\mathrm{~d}, 1 \mathrm{H}, J=11.6 \mathrm{~Hz}), 4.69(\mathrm{~m}, 2 \mathrm{H}), 6.87(\mathrm{~d}, 2 \mathrm{H}, J=8.8 \mathrm{~Hz}) 7.28(\mathrm{~d}, 2 \mathrm{H}, J=8.8$ $\mathrm{Hz}) .{ }^{13} \mathrm{C} \mathrm{NMR}\left(100 \mathrm{MHz}, \mathrm{CDCl}_{3}\right) \delta 19.8,25.9,31.0,55.6,62.4,68.8,97.7,113.9,129.6$, $130.5,159.2$.

5a. ${ }^{1} \mathrm{H}$ NMR $\left(400 \mathrm{MHz}, \mathrm{CDCl}_{3}\right) \delta 1.43-1.99(\mathrm{~m}, 6 \mathrm{H}), 3.52(\mathrm{~m}, 1 \mathrm{H}), 3.89(\mathrm{~m}, 1 \mathrm{H}), 4.75(\mathrm{~m}$, $1 \mathrm{H}), 4.89(\mathrm{~d}, 1 \mathrm{H}, J=15.6 \mathrm{~Hz}), 5.15(\mathrm{~d}, 1 \mathrm{H}, J=14.8 \mathrm{~Hz}), 7.40(\mathrm{t}, 1 \mathrm{H}, J=7.2 \mathrm{~Hz}), 7.63(\mathrm{t}$, $1 \mathrm{H}, J=7.2 \mathrm{~Hz}), 7.80(\mathrm{~d}, 1 \mathrm{H}, J=7.6 \mathrm{~Hz}), 8.05(\mathrm{~d}, 1 \mathrm{H}, J=8.0 \mathrm{~Hz}) .{ }^{13} \mathrm{C} \mathrm{NMR}(100 \mathrm{MHz}$, 
$\left.\mathrm{CDCl}_{3}\right) \delta 19.7,25.7,30.8,62.6,66.1,98.9,124.7,128.0,128.9,133.6,135.2,147.4$. Peaks from other enantiomers $(20.1,25.8,31.6,63.1,94.7)$. Anal. calcd for $\mathrm{C}_{12} \mathrm{H}_{15} \mathrm{NO}_{4}: \mathrm{C}, 60.75$; H 6.37; N 5.90. Found C, 61.04, H, 6.31, N, 5.82.

6a. ${ }^{1} \mathrm{H}$ NMR (400 MHz, $\left.\mathrm{CDCl}_{3}\right) \square \delta 1.48-2.0(\mathrm{~m}, 6 \mathrm{H}), 3.55(\mathrm{~m}, 1 \mathrm{H}), 3.88(\mathrm{~m}, 1 \mathrm{H}), 4.50(\mathrm{~d}$, $1 \mathrm{H}, J=13.2 \mathrm{~Hz}), 4.79(\mathrm{~m}, 2 \mathrm{H}), 4.80(\mathrm{~d}, 1 \mathrm{H}, J=13.2 \mathrm{~Hz}), 7.23(\mathrm{~m}, 1 \mathrm{H}), 7.35(\mathrm{~d}, 1 \mathrm{H}, J=1.6$ $\mathrm{Hz}), 7.44(\mathrm{~d}, 1 \mathrm{H}, J=8.4 \mathrm{~Hz}) .{ }^{13} \mathrm{C} \mathrm{NMR}\left(100 \mathrm{MHz}, \mathrm{CDCl}_{3}\right) \delta 19.7,25.8,30.8,62.5,66.0$, 98.6, 127.1, 129.1, 129.8, 133.5, 133.6, 135.0.

7a. ${ }^{1} \mathrm{H}$ NMR $\left(400 \mathrm{MHz}, \mathrm{CDCl}_{3}\right) \delta 1.40-1.98(\mathrm{~m}, 9 \mathrm{H}), 3.50(\mathrm{~m}, 1 \mathrm{H}), 3.98(\mathrm{~m}, 1 \mathrm{H}), 4.40(\mathrm{~m}$, $1 \mathrm{H}), 4.90(\mathrm{~m}, 1 \mathrm{H}), 7.30(\mathrm{~m}, 5 \mathrm{H}) .{ }^{13} \mathrm{C} \mathrm{NMR}\left(100 \mathrm{MHz}, \mathrm{CDCl}_{3}\right) \delta 20.2,24.8,25.98,31.2$, 63.0, 73.5, 96.3, 126.6, 127.1, 128.3, 143.8. Peaks from other diastereomers $(19.7,22.4$, 25.95, 31.3, 62.2, 73.3, 96.4, 126.1, 127.5, 128.5, 143.9). Anal. calcd for $\mathrm{C}_{13} \mathrm{H}_{18} \mathrm{O}_{2} \mathrm{C}, 75.69$; H 8.80. Found C, 75.78, H, 8.92.

8a. ${ }^{1} \mathrm{H}$ NMR $\left(400 \mathrm{MHz}, \mathrm{CDCl}_{3}\right)$ Obtained as a mixture of diastereomers. ${ }^{13} \mathrm{C}$ NMR (100 $\mathrm{MHz}, \mathrm{CDCl}_{3}$ ) (Peaks from both diastereomers) $\delta 16.0,16.7,20.1,20.7,21.6,21.7,22.7$, 22.8, 23.4, 23.7, 25.6, 25.9, 26.00, 26.04, 31.6, 31.7, 31.8, 32.2, 34.8, 34.9, 40.5, 43.9, 48.5, 49.2, 62.7, 63.3, 74.4, 80.2, 94.6, 101.5. Anal. calcd for $\mathrm{C}_{15} \mathrm{H}_{28} \mathrm{O}_{2}$ : C, 74.95; H 11.74. Found $\mathrm{C}, 74.58, \mathrm{H}, 12.02$.

9a. ${ }^{1} \mathrm{H}$ NMR $\left(400 \mathrm{MHz}, \mathrm{CDCl}_{3}\right)$ Obtained as a mixture of diastereomers $\delta 0.93(\mathrm{t}, 3 \mathrm{H}, J=7.6$ $\mathrm{Hz}), 1.41-1.88(\mathrm{~m}, 8 \mathrm{H}), 3.48(\mathrm{~m}, 1 \mathrm{H}), 3.95(\mathrm{~m}, 1 \mathrm{H}), 4.40(\mathrm{t}, 1 \mathrm{H}, J=4 \mathrm{~Hz}), 4.59(\mathrm{t}, 1 \mathrm{H}, J=6$ $\mathrm{Hz}), 7.2-7.4(\mathrm{~m}, 5 \mathrm{H}) .{ }^{13} \mathrm{C} \mathrm{NMR}\left(100 \mathrm{MHz}, \mathrm{CDCl}_{3}\right) \delta 11.0,20.0,26.0,31.2,31.6,62.7,78.8$, 95.5, 126.7, 127.2, 128.2, 142.5, Peaks from other diastereomers $(10.2,19.6,25.9,30.1,31.1$, 62.2, 80.1, 98.1, 127.0, 127.5, 128.4, 143.5). Anal. calcd for $\mathrm{C}_{14} \mathrm{H}_{20} \mathrm{O}_{2}$ : C, 76.33; H 9.15. Found C, 76.59, H, 9.04.

10a. ${ }^{1} \mathrm{H}$ NMR (400 MHz, $\left.\mathrm{CDCl}_{3}\right) \delta 1.50-1.86(\mathrm{~m}, 6 \mathrm{H}), 3.50(\mathrm{~m}, 1 \mathrm{H}), 3.90(\mathrm{~m}, 1 \mathrm{H}), 4.15(\mathrm{~m}$, $1 \mathrm{H}), 4.38(\mathrm{~m}, 1 \mathrm{H}), 4.69(\mathrm{~m}, 1 \mathrm{H}), 6.30(\mathrm{~m}, 1 \mathrm{H}), 6.60(\mathrm{~d}, 1 \mathrm{H}, J=16 \mathrm{~Hz}), 7.15-7.40(\mathrm{~m}, 5 \mathrm{H})$. ${ }^{13} \mathrm{C}$ NMR $\left(100 \mathrm{MHz}, \mathrm{CDCl}_{3}\right) \delta 19.9,25.9,31.0,62.5,67.9,98.1,126.2,126.7,127.8,128.7$, $132.5,136.9$.

11a. ${ }^{1} \mathrm{H}$ NMR $\left(400 \mathrm{MHz}, \mathrm{CDCl}_{3}\right) \delta 1.50-2.0$ (brm, $\left.6 \mathrm{H}\right), 3.45(\mathrm{~m}, 1 \mathrm{H}), 3.54(\mathrm{t}, 1 \mathrm{H}, J=8.8$ $\mathrm{Hz}), 3.83(\mathrm{~m}, 1 \mathrm{H}), 4.05(\mathrm{t}, 1 \mathrm{H}, J=8.0 \mathrm{~Hz}), 4.18(\mathrm{t}, 1 \mathrm{H}, J=8.0 \mathrm{~Hz}), 4.67(\mathrm{t}, 1 \mathrm{H}, J=3.2 \mathrm{~Hz})$, $7.26(\mathrm{~d}, 2 \mathrm{H}, J=7.6 \mathrm{~Hz}), 7.34(\mathrm{t}, 2 \mathrm{H}, J=7.2 \mathrm{~Hz}), 7.63(\mathrm{~d}, 2 \mathrm{H}, J=7.6 \mathrm{~Hz}), 7.72(\mathrm{~d}, 2 \mathrm{H}, J=7.2$ $\mathrm{Hz}) .{ }^{13} \mathrm{C} \mathrm{NMR}\left(100 \mathrm{MHz}, \mathrm{CDCl}_{3}\right) \delta 18.0,24.1,29.2,46.4,60.5,68.6,97.4,118.17,118.18$, $123.6,123.7,125.2,125.2,125.7,139.5,143.0,143.5$.

12a. ${ }^{1} \mathrm{H}$ NMR (400 MHz, $\mathrm{CDCl}_{3}$ ) Obtained as a mixture of diastereomers $\delta 1.30-1.84$ (brm, $12 \mathrm{H}), 3.49(\mathrm{~m}, 2 \mathrm{H}), 3.75(\mathrm{~m}, 3 \mathrm{H}), 4.06(\mathrm{~m}, 1 \mathrm{H}), 4.29(\mathrm{~m}, 1 \mathrm{H}), 4.62(\mathrm{~m}, 1 \mathrm{H}) .{ }^{13} \mathrm{C}$ NMR $(100$ $\mathrm{MHz}, \mathrm{CDCl}_{3}$ ) Peaks from both diastereomers $\delta$ 19.6, 19.8, 25.7, 25.8, 25.9, 27.0, 27.1, 30.7, $62.3,62.5,67.0,67.2,68.2,68.8,74.9,75.1,99.1,99.3,109.4,109.6$; Anal. calcd for $\mathrm{C}_{11} \mathrm{H}_{20} \mathrm{O}_{4}$ : C, 61.09; $\mathrm{H}$ 9.32. Found C, 61.23, H, 8.43.

13a. ${ }^{1} \mathrm{H}$ NMR (400 MHz, $\left.\mathrm{CDCl}_{3}\right) \delta 1.50-1.86(\mathrm{~m}, 6 \mathrm{H}), 3.54(\mathrm{~m}, 1 \mathrm{H}), 3.90(\mathrm{~m}, 1 \mathrm{H}), 4.42(\mathrm{~d}$, $1 \mathrm{H}, J=11.2 \mathrm{~Hz}), 4.52(\mathrm{~m}, 2 \mathrm{H}), 4.70(\mathrm{~m}, 2 \mathrm{H}), 5.30(\mathrm{dd}, 2 \mathrm{H}, J=10.8 \mathrm{~Hz}, J=17.2 \mathrm{~Hz}), 6.02$ $(\mathrm{m}, 1 \mathrm{H}), 6.87(\mathrm{~d}, 2 \mathrm{H}, J=8.0 \mathrm{~Hz}), 7.27(\mathrm{~d}, 2 \mathrm{H}, J=8.4 \mathrm{~Hz}) .{ }^{13} \mathrm{C} \mathrm{NMR}\left(100 \mathrm{MHz}, \mathrm{CDCl}_{3}\right) \delta$ 19.8, 25.8, 30.9, 62.4, 68.8, 69.1, 97.7, 114.8, 117.8, 129.6, 130.6, 133.4, 158.2.

14a. ${ }^{1} \mathrm{H}$ NMR (400 MHz, $\left.\mathrm{CDCl}_{3}\right) \delta 1.45-1.90(\mathrm{brm}, 6 \mathrm{H}), 3.51(\mathrm{~m}, 1 \mathrm{H}), 3.80(\mathrm{~m}, 1 \mathrm{H}), 3.88$ $(\mathrm{m}, 1 \mathrm{H}), 4.05(\mathrm{~m}, 1 \mathrm{H}), 4.51(\mathrm{~m}, 2 \mathrm{H}), 4.71(\mathrm{~m}, 1 \mathrm{H}), 7.44(\mathrm{~m}, 2 \mathrm{H}), 7.55(\mathrm{~m}, 1 \mathrm{H}), 8.06(\mathrm{~m}, 2 \mathrm{H})$. 
${ }^{13} \mathrm{C}$ NMR $\left(100 \mathrm{MHz}, \mathrm{CDCl}_{3}\right) \delta 19.6,25.8,30.8,62.3,64.5,65.5,99.0,128.5,129.8,130.3$, 133.0, 166.6.

15a. ${ }^{1} \mathrm{H}$ NMR (400 MHz, $\left.\mathrm{CDCl}_{3}\right) \delta 0.03(\mathrm{~s}, 6 \mathrm{H}), 0.88(\mathrm{~s}, 9 \mathrm{H}), 1.28-1.90(\mathrm{~m}, 14 \mathrm{H}), 3.37$ (m, $1 \mathrm{H}), 3.48(\mathrm{~m}, 1 \mathrm{H}), 3.59(\mathrm{t}, 2 \mathrm{H}, J=6.8 \mathrm{~Hz}), 3.70(\mathrm{~m}, 1 \mathrm{H}), 3.84(\mathrm{~m}, 1 \mathrm{H}), 4.56(\mathrm{~m}, 1 \mathrm{H}) .{ }^{13} \mathrm{C}$ NMR $\left(100 \mathrm{MHz}, \mathrm{CDCl}_{3}\right) \delta-4.7,18.7,20.0,25.9,26.1,26.3,26.4,30.1,31.1,33.2,62.5$, 63.5, 67.8, 99.0.

16a. ${ }^{1} \mathrm{H}$ NMR (400 MHz, $\left.\mathrm{CDCl}_{3}\right) \delta 1.40-1.88(\mathrm{~m}, 20 \mathrm{H}), 3.38(\mathrm{~m}, 2 \mathrm{H}), 3.48(\mathrm{~m}, 2 \mathrm{H}), 3.73(\mathrm{~m}$, $2 \mathrm{H}), 3.85(\mathrm{~m}, 2 \mathrm{H}), 4.57(\mathrm{~m}, 2 \mathrm{H}) .{ }^{13} \mathrm{C} \mathrm{NMR}\left(100 \mathrm{MHz}, \mathrm{CDCl}_{3}\right) \delta 20.1,25.9,26.5,30.1,31.1$, $62.6,67.8,99.0$.

17a. ${ }^{1} \mathrm{H}$ NMR (400 MHz, $\left.\mathrm{CDCl}_{3}\right) \delta 1.22-1.83(\mathrm{~m}, 14 \mathrm{H}), 2.45(\mathrm{~s}, 3 \mathrm{H}), 3.34(\mathrm{~m}, 1 \mathrm{H}), 3.49(\mathrm{~m}$, $1 \mathrm{H}), 3.68(\mathrm{~m}, 1 \mathrm{H}), 3.83(\mathrm{~m}, 1 \mathrm{H}), 4.02(\mathrm{t}, 2 \mathrm{H}, J=6.4 \mathrm{~Hz}), 4.54(\mathrm{~m}, 1 \mathrm{H}), 7.33(\mathrm{~d}, 2 \mathrm{H}, J=8.4$ $\mathrm{Hz}), 7.77(\mathrm{~d}, 2 \mathrm{H}, J=8.4 \mathrm{~Hz}) .{ }^{13} \mathrm{C} \mathrm{NMR}\left(100 \mathrm{MHz}, \mathrm{CDCl}_{3}\right) \delta 20.1,22.0,25.6,25.8,26.0$, 29.1, 29.8, 31.1, 62.7, 67.6, 70.8, 99.1, 128.0, 129.9, 133.3, 144.7 .

18a. ${ }^{1} \mathrm{H}$ NMR (400 MHz, $\left.\mathrm{CDCl}_{3}\right) \delta 1.40-1.84(\mathrm{brm}, 13 \mathrm{H}), 3.43(\mathrm{~m}, 1 \mathrm{H}), 3.50(\mathrm{~m}, 1 \mathrm{H}), 3.65$ $(\mathrm{t}, 2 \mathrm{H}, J=6.4 \mathrm{~Hz}), 3.75(\mathrm{~m}, 1 \mathrm{H}), 3.84(\mathrm{~m}, 1 \mathrm{H}), 4.57(\mathrm{~m}, 1 \mathrm{H}) ;{ }^{13} \mathrm{C} \mathrm{NMR}\left(100 \mathrm{MHz}, \mathrm{CDCl}_{3}\right) \delta$ 20.0, 22.8, 25.8, 29.8, 31.1, 32.9, 62.7, 63.1, 67.8, 99.1.

18b. ${ }^{1} \mathrm{H}$ NMR (400 MHz, $\left.\mathrm{CDCl}_{3}\right) \delta 1.44-1.86(\mathrm{~m}, 18 \mathrm{H}), 3.39(\mathrm{~m}, 2 \mathrm{H}), 3.49(\mathrm{~m}, 2 \mathrm{H}), 3.72$ $(\mathrm{m}, 2 \mathrm{H}), 3.86(\mathrm{~m}, 2 \mathrm{H}), 4.57(\mathrm{~m}, 2 \mathrm{H}) ;{ }^{13} \mathrm{C}$ NMR $\left(100 \mathrm{MHz}, \mathrm{CDCl}_{3}\right) \delta$ 20.1, 23.3, 25.9, 30.0, 31.1, 62.6, 67.8, 99.0.

16. ${ }^{1} \mathrm{H}$ NMR $\left(400 \mathrm{MHz}, \mathrm{CDCl}_{3}\right) \delta 1.39(\mathrm{~m}, 4 \mathrm{H}), 1.50-1.80(\mathrm{~m}, 11 \mathrm{H}), 3.38(\mathrm{~m}, 1 \mathrm{H}), 3.49(\mathrm{~m}$, $1 \mathrm{H}), 3.63(\mathrm{t}, 2 \mathrm{H}, J=6.4 \mathrm{~Hz}), 3.74(\mathrm{~m}, 1 \mathrm{H}), 3.85(\mathrm{~m}, 1 \mathrm{H}), 4.57(\mathrm{~m}, 1 \mathrm{H}) .{ }^{13} \mathrm{C}$ NMR $(100 \mathrm{MHz}$, $\left.\mathrm{CDCl}_{3}\right) \delta 20.0,25.8,25.9,26.4,30.0,31.1,33.0,62.6,62.9,67.8,99.0$.

20a. ${ }^{1} \mathrm{H}$ NMR (400 MHz, $\left.\mathrm{CDCl}_{3}\right) \delta 1.23(\mathrm{~d}, 3 \mathrm{H}, J=6.4 \mathrm{~Hz}), 1.40-1.68(\mathrm{~m}, 9 \mathrm{H}), 2.09$ (brs, $1 \mathrm{H}), 3.62(\mathrm{t}, 2 \mathrm{H}, J=6.4 \mathrm{~Hz}), 3.73(\mathrm{~m}, 2 \mathrm{H}), 4.07(\mathrm{~m}, 1 \mathrm{H}), 4.53(\mathrm{t}, 1 \mathrm{H}, J=5.2 \mathrm{~Hz}) .{ }^{13} \mathrm{C}$ NMR $\left(100 \mathrm{MHz}, \mathrm{CDCl}_{3}\right) \delta$ 20.6, 22.0, 32.6, 33.3, 34.9, 62.4, 66.7, 73.0, 102.0.

20b. ${ }^{1} \mathrm{H}$ NMR ( $400 \mathrm{MHz}, \mathrm{CDCl}_{3}$ ) Obtained as a mixture of diastereomers $\delta 1.23(\mathrm{~d}, 3 \mathrm{H}, J=$ $6.4 \mathrm{~Hz}), 1.40-1.84(\mathrm{~m}, 14 \mathrm{H}), 3.37(\mathrm{~m}, 1 \mathrm{H}), 3.49(\mathrm{~m}, 1 \mathrm{H}), 3.71(\mathrm{~m}, 3 \mathrm{H}), 3.85(\mathrm{~m}, 1 \mathrm{H}), 4.06$ $(\mathrm{m}, 1 \mathrm{H}), 4.52(\mathrm{~m}, 2 \mathrm{H}) .{ }^{13} \mathrm{C}$ NMR $\left(100 \mathrm{MHz}, \mathrm{CDCl}_{3}\right)$ Peaks from both diastereomers $\delta 19.8$, 20.0, 21.1, 21.2, 21.9, 22.1, 25.7, 25.8, 29.8, 29.9, 30.95, 31.09, 33.2, 33.4, 35.1, 35.3, 62.2, 62.5, 66.6, 66.8, 67.4, 67.6, 72.7, 72.9, 98.7, 98.9, 101.8, 102.0. Anal. calcd for $\mathrm{C}_{14} \mathrm{H}_{26} \mathrm{O}_{4}$ : C, 65.09; H 10.14. Found C, 65.26, H, 9.87.

21a. ${ }^{1} \mathrm{H}$ NMR $\left(400 \mathrm{MHz}, \mathrm{CDCl}_{3}\right) \delta 1.48-1.84(\mathrm{~m}, 6 \mathrm{H}), 2.82(\mathrm{t}, 2 \mathrm{H}, J=12 \mathrm{~Hz}), 3.48-3.62(\mathrm{~m}$, 2H), 3.79-3.92 (m, 2H), $4.61(\mathrm{~m}, 1 \mathrm{H}), 6.07$ (brs, $1 \mathrm{H}), 6.73$ (d, 2H, $J=8.4 \mathrm{~Hz}$,), $7.05(\mathrm{~d}, 2 \mathrm{H}, J$ $=8.4 \mathrm{~Hz}) .{ }^{13} \mathrm{C}$ NMR $\left(100 \mathrm{MHz}, \mathrm{CDCl}_{3}\right) \delta 19.4,25.4,26.4,30.6,35.4,62.2$, 68.6, 98.7, 115.1, 130.0, 130.7, 154.3. 